\title{
Optimizing Online Sequential Extreme Learning Machine Parameters and Application to Transformer Fault Diagnosis
}

\author{
Wenrong Kang ${ }^{1, a}$, Wenyan Chen ${ }^{2, b}$ \\ ${ }^{1}$ School of electrical And engineering control, Xi an University of Science and Technology Graduate \\ School, xi'an , 10704,China \\ ${ }^{2}$ School of electrical And engineering control, Xi'an University of Science and Technology Graduate \\ School, xi'an , 10704,China \\ aemail: 541397020@qq.com, bemail:ch_w_y@163.com
}

Keywords: Online Sequential; extreme learning machine; Genetic Algorithm Optimization; powers transformer fault diagnosis; parameter optimization

\begin{abstract}
In order to solve the problem that the (OS-ELM) is used in the fault diagnosis of the transformer, the genetic algorithm (Algorithm Genetic) is applied to the on-line extreme learning machine, and a new method of transformer fault diagnosis is proposed. In this method, the number of hidden layer neurons of the Block L, the data set size $\mathrm{N}$, and the hidden layer activation function are selected by the Algorithm Genetic optimization algorithm. Through simulation test, the fault diagnosis of transformer is $99.56 \%$, and the test time is $0.0024 \mathrm{~s}$. Compared with the optimization, the diagnostic accuracy and the test time of the transformer fault are improved obviously.
\end{abstract}

\section{Introduction}

In the long run,the transformer will inevitably lead to failure, Using the historical data and current status data of transformer fault to analyze and identify the fault to eliminate potential failures and defects as soon as possible, has been the concern of researchers study.

There were many traditional transformer fault diagnosis intelligent methods,for example, IEC recommended three ratio method, Rogers and Dornerburg method etc. But coding boundary is too absolute and coding need be artificially drawn in the traditional threshold detection means. There is some defects especially lack of encoding[1][2]; At the same time, emerging intelligent methods such as BP neural network, SVM,Bayesian network and so on, are facing the main problems example high intensity human interference, learning speed slowly, poor learning extensibility and a large sample of demand etc[5].The existing DGA data cannot meet the demand of their training[3][6].When applied to the high response demanding online program, these emerging intelligent methods are often inefficient[4].

At present, the research of the online sequence extreme learning machine (OS-ELM) is used in the diagnosis of transformer fault[7], and the data are effectively avoided,However, the artificial choice of the online sequence of extreme learning machine parameters, resulting in the limit of learning machine training accuracy is not high enough.

Genetic algorithm is proposed to optimize the fault diagnosis method of OS-ELM, and the parameters of the machine parameters are optimized by using genetic algorithm to optimize the parameters of OS-ELM[8][9].The feasibility and superiority of the proposed method is verified by the comparison of the training accuracy and training time.

\section{Introduction of OS-ELM}

Online sequence extreme learning machine (OS-ELM) is a single hidden layer forward neural network (SLFN) training algorithm which can be applied to some regression and classification tasks. The method adopts the way of partitioned matrix effectively to avoid the repeated training data and to a great extent improve the efficiency of learning.

OS-ELM algorithm described as follows: 
Given training data set $N$,the hidden layer output function $G\left(a_{i}, b_{i}, x\right)$ and the number of hidden layer nodes $L$.

Step1 Initialization phase: choose the part of the data set $N_{0}=\left\{x_{i}, t_{i}\right\}_{i=1}^{N_{0}}$ form the $N$, where $N_{0} \geqslant L$

a)Randomly selected input weights $a_{i}$ and threshold of hidden layer node $b_{i, i} i=1, \ldots L$;

b)Calculate the output matrix In the hidden layer $H_{0}$

$$
H_{0}=\left[\begin{array}{ccc}
G\left(\mathrm{a}_{1}, \mathrm{~b}_{1}, \mathrm{x}_{1}\right) & \cdots & G\left(\mathrm{a}_{L}, \mathrm{~b}_{L}, \mathrm{x}_{1}\right) \\
G\left(\mathrm{a}_{1}, \mathrm{~b}_{1}, \mathrm{x}_{2}\right) & \cdots & G\left(\mathrm{a}_{L}, \mathrm{~b}_{L}, \mathrm{x}_{2}\right) \\
\vdots & & \vdots \\
G\left(\mathrm{a}_{1}, \mathrm{~b}_{1}, \mathrm{x}_{N_{0}}\right) & \cdots & G\left(\mathrm{a}_{L}, \mathrm{~b}_{L}, \mathrm{x}_{N_{0}}\right)
\end{array}\right]_{N_{0} \times L}
$$

c) Calculate the initial output weights $\beta^{0}=P_{0} H_{0}^{T} T_{0}$, where $P_{0}=\left(\mathrm{H}_{0}^{T} \mathrm{H}_{0}\right)^{-1}$

d)make $K=0$

Step 2 Sequence learning phase: set the $k+1$ step to add the data block as $N_{k+1}=\left\{\left(x_{i}, t_{i}\right)\right\}_{i=\left(\sum_{j=0}^{k} N_{j}\right)+1}^{\substack{k+1 \\ j}}$.

a) Calculate output matrix of the hidden layer $H_{k+1}$, after the new data to add.

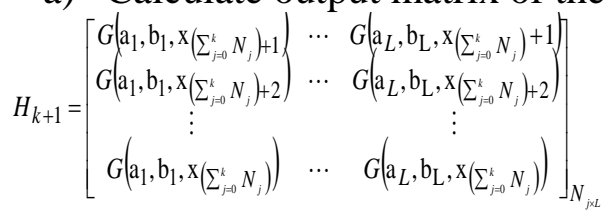

make $\mathbf{T}_{k+1}=\left(\boldsymbol{t}_{\left(\sum_{j=0}^{k} N_{f}\right)+1}, \cdots, \boldsymbol{t}_{\left(\sum_{j=0}^{k+1} N_{f}\right)}\right)^{\mathrm{T}}$

b) to calculate the output weights $\beta^{k+1}$.

$$
\begin{aligned}
& \beta^{k+1}=\beta^{K}+P_{k} H_{k=1}^{T}\left(T_{k+1}-H_{k+1} \beta^{k}\right) \\
& P_{k+1}=P_{k}-P_{k} H_{k+1}^{T}\left(I+H_{k+1} P_{k} H_{k+1}^{T}\right)^{-1} H_{k+1} P_{k}
\end{aligned}
$$

c) make $k=k+1$, return to Step 2 .

Therefore, the OS-ELM algorithm needs to set the parameters of the hidden layer neuron number $L$, the size of the data set $N_{0}$, and the size of the data blocks in the learning process Block artificially. The setting of these parameters is an important factor to affect the performance of OS-ELM classifier.

\section{The transformer fault diagnosis based on OS-ELM and Genetic algorithm}

From the last chapter we can know,the accuracy of OS-ELM algorithm is related to the initial setting of parameters, and different parameters have different effects on the accuracy of OS-ELM classifier[10][11]. The parameters of artificial setting are not high, so the parameters are optimized by genetic algorithm.

The genetic algorithm is used to optimize the parameters of OS-ELM classifier can be divided into the following steps, flow chart, as shown in Figure 1.

Step 1: processing the sample data first, randomly generated weights and thresholds;

Step 2: calculate the fitness value, and the roulette wheel selection OS-ELM classifier parameters;

Step 3: determine whether to meet the termination conditions, if satisfied, the steering step 5, if not satisfied, turn to step 4;

Step 4: the generation of the next generation of hybrid variation, to step 2;

Step 5: output optimal parameters and optimal model.

Genetic algorithm is a global optimization algorithm, which can overcome the shortcomings of the traditional iterative method in the dead cycle, through the selection, crossover and mutation of the three basic operators to obtain the optimal solution of the parameters. The fault diagnosis of 
power transformer by put the optimization parameter into the OS-ELM algorithm.

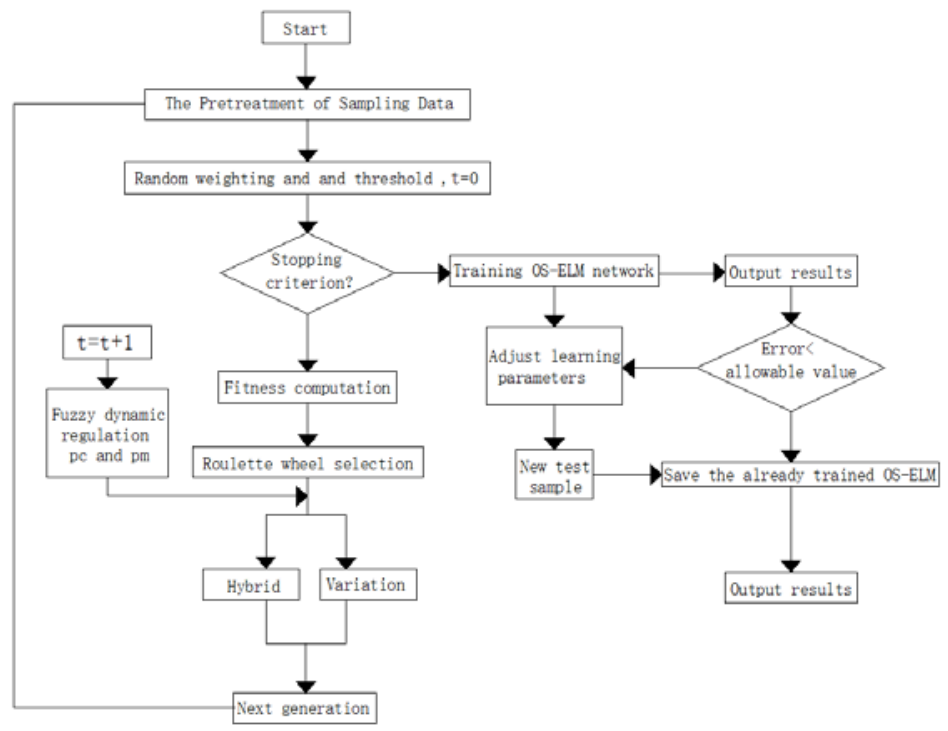

Fig.1. Flow chart of GA-oselm

\section{Choice of the characteristics.}

This article selects IEC recommended DGA data $\mathrm{H}_{2}, \mathrm{CH}_{4}, \mathrm{C}_{2} \mathrm{H}_{6}, \mathrm{C}_{2} \mathrm{H}_{4}$ and $\mathrm{C}_{2} \mathrm{H}_{2}$ five gases dissolved amount as the input of OS-ELM algorithm.Since the DGA data value distribution range is very large, even though there may be larger differences between the same type of data, in order to reduce its influence due to differences in values between each other. Before the characteristics are input in classifier, data must be normalized to process according to formula.

$$
X_{\text {normalized }}=L o+\frac{x-x_{\min }}{x_{\max }-x_{\min }}(U p-L o)
$$

In the type: $x_{\text {normalized }}$ is a gas concentration values after data is normalized to process. $x_{\text {min }}$ is Minimum gas content. $x_{\max }$ is Maximum gas content. $U_{p}$ and $L_{o}$ are respectively upper bound and lower bound of the normalization and respectively value 1 and -1 .

\section{The gas sample selection and transformer status code.}

In transformer DGA data, the fault samples belong to minority class samples. In the original data has two unbalanced phenomenon: the unbalanced phenomenon between normal samples and fault samples and the unbalanced phenomenon between the various fault samples. We should select samples to keep a balance of samples. In this experiment, the data collection of HengShui city, HeBei province power bureau and other relevant literature of the 268 groups of data, according to the proportion of 3:1 is divided into training samples and testing samples. All sample sizes are shown in Table 1. 
Table.1.Transformers state code table

\begin{tabular}{ccc}
\hline Status Category & Number & State vector \\
\hline $\begin{array}{c}\text { Normal (N) } \\
\text { Partial Discharge } \\
\text { (PD) }\end{array}$ & 28 & $(0,0,0,0,0,1)$ \\
Low energy & 30 & $(0,0,0,0,1,0)$ \\
discharge (D1) & 19 & $(0,0,0,1,0,0)$ \\
$\quad \begin{array}{l}\text { High energy } \\
\text { discharge (D2) }\end{array}$ & 32 & $(0,0,1,0,0,0)$ \\
$\begin{array}{c}\text { Low temperature } \\
\text { thermal (T12) }\end{array}$ & 34 & $(0,1,0,0,0,0)$ \\
Super heater (T3) & 40 & $(1,0,0,0,0,0)$ \\
\hline
\end{tabular}

Because the transformer fault diagnosis is more than a classification task and OS-ELM algorithm has more classification ability, a classifier transformer status categories can divided six kinds of state into normal, partial discharge, low discharge, overheating in low-temperature, high temperature and high energy discharge superheat.OS-ELM output is defined as a vector classification and classification of vector dimension is defined as the number of samples status categories. This algorithm should code for each state in the application. As shown in the Table 1.

\section{The selection of OS-ELM and Genetic algorithm algorithm parameters.}

OS-ELM algorithm needs to set the parameters of the human, the choice of different parameters will affect the accuracy of the algorithm. But in the implementation of the GA-oselm algorithm, these parameters do not need manual settings. Because the genetic algorithm simulates natural selection and natural genetic processes in replication, crossover and mutation process, a set of candidate solutions is retained in each iteration[13][14]. The genetic operators (selection, crossover and mutation) are used to generate a new generation of candidate solutions.

According to he parameters of OS-ELM algorithm are optimized by genetic algorithm,we should set the number $L, N_{0}$ and Block of hidden layer neurons parameter. Set $L=100$. $N_{0}$ amount should not be less than the number of hidden layer neurons $L$, so $N_{0}=176, B l o c k=5$, and we should select activation functions Hardlim function.

\section{The realization of the transformer fault diagnosis based on OS-ELM and Genetic algorithm.}

In this paper, the experiment run in the Intel (R) Core (TM) 2 Duo CPU P7350 2.00GHz, 2.00 GB of system memory hardware environment, the operating system to Windows 732 bit and on Matlab R2013a version. Data is collected a total of 268 sets by HengShui city, HeBei province power bureau and other relevant documents data. In order to verify the effectiveness of the proposed method, we choose the same parameters $L, N_{0}$, Block and activation function Hardlim function.Genetic algorithm optimization OS-ELM training in transformer fault diagnosis accuracy as shown in Figure 2, with genetic algorithm generations of selection, crossover and mutation gradually get the optimal solution, optimization parameters online the limit of a sequence of machine learning to transformer fault diagnosis accuracy increased gradually. 


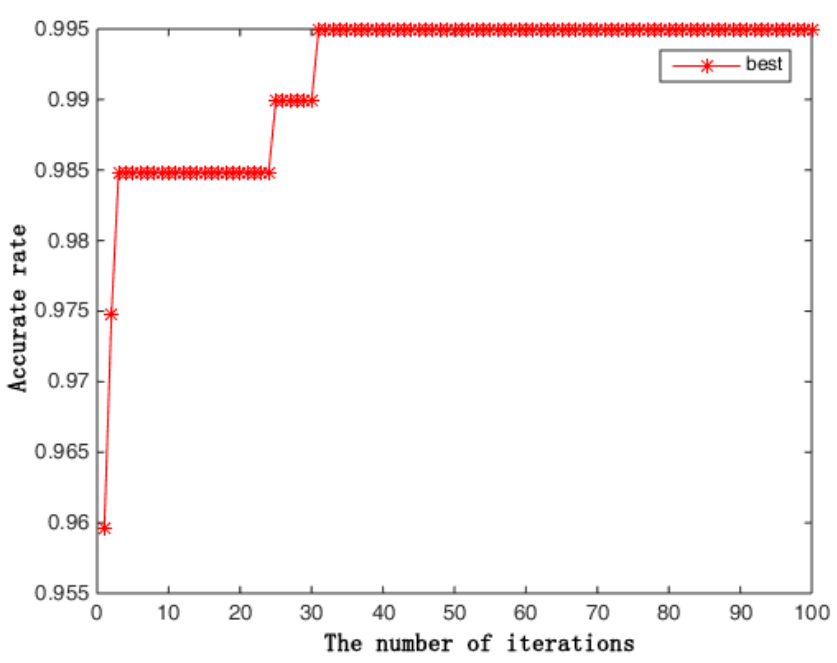

Fig.2. the GA-os-elm accuracy comparation

Compared with the simulation results of several common algorithms such as training time and test time and accuracy, it can be seen that the optimization of genetic algorithm is better than SVM, ELM and OS-ELM in terms of training time, test time and accuracy.

Table.2.the SVM, ELM, OS-ELM algorithm comparison

\begin{tabular}{ccccc}
\hline Algorithm & Parameters & Training time/s & Testing time/s & Accuracy\% \\
\hline SVM & $\begin{array}{c}\mathrm{C}=500 \\
=0.5\end{array}$ & 0.5547 & 0.0125 & 84.35 \\
\hline ELM & $\mathrm{L}=100$ & 0.0624 & 0.0178 & 85.46 \\
\hline OS-ELM & $\begin{array}{r}\mathrm{L}=100 \mathrm{~N}_{\mathrm{O}}=160 \\
\text { Block=10 }\end{array}$ & 0.0451 & 0.0053 & 86.37 \\
\hline GA-OSELM & $\begin{array}{l}\mathrm{N}_{\mathrm{O}}=176 \\
\text { Block=5 }\end{array}$ & 0.0134 & 0.0024 & 99.56 \\
\hline
\end{tabular}

\section{Conclusion.}

In this paper, we present a new method for transformer fault diagnosis, This approach is the use of genetic algorithm to optimize the parameters of the OS-ELM algorithm.No optimized OS-ELM algorithm can only use artificial methods to select parameters and activation functions,OS-ELM is optimized by genetic algorithm, which is based on a single iteration, and the training accuracy is more obvious. After Matlab simulation,analysis and comparison of SVM, OS-ELM, ELM and GA-OS-ELM.The advantages of GA-OS-ELM in training time, test time and accuracy are more obvious. The results show that the genetic algorithm optimization of OS-ELM has a faster response speed and higher efficiency in transformer fault on-line monitoring and on-line diagnosis.

\section{References}

[1] P Mirowski, Y LeCun. Statistical Machine Learning and Dissolved Gas Analysis: A Review [J].IEEE Transactions on Power Delivery,2010,27(4):1-6.

[2] Ronaldo R.B.de Aquino, Milde M.S.Lira.A fuzzy system for detection of incipient fault in power transformers based on gas-in-oil analysis[C]. Fuzzy Systems (FUZZ), 2010 IEEE International Conference on,2010.7:1-6.

[3] Du Wenxia,Lv Feng,Ju Xiyuan, et al. Fault Diagnosis of Power Transformer Based on BP Neural Network[J]. Transformer, 2007,44(3):45-47.

[4] Li Shuang,Wang Langzhu,Zhang Wei,et al.Fault Diagnosis Method of Transformer Based on Improved BP Neural Network of DGA[J]. Transformer, 2010,47(12):61-65.

[5] Zhao Wenqing,Zhu Yongli,Wang Xiaohui. Fault Diagnosis of Power Transformer Based on 
Combination of Bayesian Networks[J].Electric Power Automation Equipment, 2009,29(11):6-9.

[6] Li Chunxiang,Zhang Weimin,Zhong Biliang,et al.Reserach On Parameter Optimization Algorithm of Least Squares Support Vector Machine[J].Journal of Hangzhou Dianzi University, 2010, 30(4):213-216. DOI:10.3969/j.issn. 1001-9146.2010.0406

[7] Huang G B,Zhu Q Y,Siew C K.Extreme Leraning Maching:Theory and Application[J].Neurocomputing,2006,70(1/2/3):489-501.

[8] Huang G B,Zhu Q Y,Siew C K. Extreme Learning Machine:A New Learning Scheme of Feedforward Neural Networks[C].Proceedings of the International Joint Conference on Neural Networks.Piscataway:Institute of Electrical and Electronics Engineers Inc,2004:985-990.

[9] Liang Nanying,Huang Guang-bin,SaratchandranP,et al.A fast and accurate online sequential learning algorithm for feedforward networks[J].IEEE Transaction on Neural Networks,2006,17(6):1411-1423.

[10]Lan Y,Soh Y C,Huang G B. Ensemble of sequential extreme learning machine [J] .Neural Computation,2009,72:3391-3395.

[11]Zhao J W,Wang Z H,Park D S.Online sequential extreme learning machine with forgetting mechanism[J]. Neural Computation, 2012, 87: 79-89.

[12]Huynh H T,Won Y. Regularized online sequential learning algorithm for single-hidden layer feedforward neural networks[J].Pattern Recognition Letters,2011,32:1930-1935.

[13]Hoang $\mathrm{M} T$ T,Huynh $\mathrm{H}$ T,Vo $\mathrm{N}$ H,et,al.A robust online sequential extreme learning machine[J].LNCS,2007,4491:1077-1086.

[14]Yuan Lan,Yeng Chai Soh,Guang-Bin Huang. Ensemble of online sequential extreme learning machine[J].Neurocomputing,2009,72(13/14/15): 3391-3395. 